\title{
Alternative Proof of a Theorem in Change of Axes.
}

By Professor $\boldsymbol{J}_{\mathrm{ACK}}$.

If by any change of axes $a x^{2}+2 h x y+b y^{2}$ be changed into $a^{\prime} x^{\prime 2}+2 h^{\prime} x^{\prime} y^{\prime}+b^{\prime} y^{\prime 2}$, then will

$$
\begin{aligned}
\frac{a+b-2 h \cos \omega}{\sin ^{2} \omega} & =\frac{a^{\prime \prime}+b^{\prime}-2 h^{\prime} \cos \omega^{\prime}}{\sin ^{2} \omega^{\prime}}, \\
\text { and } \quad \frac{a b-h^{2}}{\sin ^{2} \omega} & =\frac{a^{\prime} b^{\prime}-h^{\prime 2}}{\sin ^{2} \omega^{\prime}} .
\end{aligned}
$$

Figure 3.

Let $O X, O Y$ be first axes ; $O X^{\prime}, O Y^{\prime}$ second axes ;

$$
\angle \mathrm{XOY}=\omega, \quad \angle \mathrm{X}^{\prime} O Y^{\prime}=\omega^{\prime} .
$$

Take $\mathrm{OA}$ a fixed line in the plane.

$$
\begin{aligned}
& \text { Iret } \angle \mathrm{AOP}=\theta \text {. } \\
& \text { Let }-\mathrm{AOX}=a \text {, } \\
& \therefore \quad \angle \mathrm{MOP}=\theta-a, \quad \angle \mathrm{M}^{\prime} \mathrm{OP}=\theta-a^{\prime} \text {; } \\
& \text { and } \quad \angle \mathrm{OPM}=\omega-(\theta-a)=\omega-\theta+a, \quad \angle \mathrm{OPM}^{\prime}=\omega^{\prime}-\theta+\alpha^{\prime} \text {. }
\end{aligned}
$$

Let $\mathrm{OP}=r$ (same in both cases).

$$
\begin{aligned}
& \text { Now } \quad \frac{\mathrm{OP}}{\sin \omega}=\frac{\mathrm{OM}}{\sin (\omega-\theta+a)}=\frac{\mathrm{MP}}{\sin (\theta-a)} ; \\
& \therefore \quad x=r \frac{\sin (\omega-\theta+a)}{\sin \omega} \text { and } y=r \frac{\sin (\theta-a)}{\sin \omega} .
\end{aligned}
$$

Similarly $x^{\prime}=r \frac{\sin \left(\omega^{\prime}-\theta+a^{\prime}\right)}{\sin \omega^{\prime}}$ and $y^{\prime}=r \frac{\sin \left(\theta-a^{\prime}\right)}{\sin \omega^{\prime}}$.

Substitute these values of $x y, x^{\prime} y^{\prime}$ in $a x^{2}+2 h x y+b y^{2}$ and $a^{\prime} x^{\prime 2}+2 h^{\prime} x^{\prime} y^{\prime}+b^{\prime} y^{\prime 2}$ respectively; then

$$
\begin{aligned}
& \frac{r^{2}}{\sin ^{2} \omega}\left\{a \sin ^{2} \overline{\omega-\theta+a}+2 h \sin \overline{\omega-\theta+\alpha} \sin \overline{\theta-\alpha}+b \sin ^{2} \overline{\theta-\alpha}\right\} \\
& \quad=\frac{r^{2}}{\sin ^{2} \omega^{\prime}}\left\{a^{\prime} \sin ^{2} \overline{\omega^{\prime}-\theta+\alpha^{\prime}}+2 h^{\prime} \sin \overline{\omega^{\prime}-\theta+a^{\prime}} \sin \overline{\theta-\alpha^{\prime}}+b^{\prime} \sin ^{2} \overline{\theta-\alpha^{\prime}}\right\}
\end{aligned}
$$

Multiply by $\frac{2}{r^{2}}$ and simplify (by trigonometry),

$$
\begin{aligned}
& \therefore \frac{1}{\sin ^{2} \omega}\{a(1-\cos \overline{2 \omega-2 \theta+2 \alpha})+2 h(\cos \overline{\omega-2 \theta+2 \alpha}-\cos \omega)+b(1-\cos \overline{2 \theta-2 \alpha})\} \\
& =\frac{1}{\sin ^{2} \omega^{\prime}}\left\{a^{\prime}\left(1-\cos 2 \overline{\omega^{\prime}-2 \theta+2 \alpha^{\prime}}\right)+2 h^{\prime}\left(\cos \overline{\omega^{\prime}-2 \theta+2 \alpha^{\prime}}-\cos \omega^{\prime}\right)+b^{\prime}\left(1-\cos \overline{2 \theta-2 \alpha^{\prime}}\right)\right\} .
\end{aligned}
$$


Expand and arrange, grouping the coefficients of $\cos 2 \theta, \sin 2 \theta$;

$$
\begin{aligned}
& \therefore \quad \frac{1}{\sin ^{2} \omega}\left\{\begin{array}{l}
a-2 h \cos \omega+b \\
-\cos 2 \theta(a \cos \overline{2 \omega+2 \alpha}-2 h \cos \overline{\omega+2 \alpha}+b \cos 2 \alpha) \\
-\sin 2 \theta(a \sin \overline{2 \omega+2 \alpha}-2 h \sin \overline{\omega+2 \alpha}+b \sin 2 a)
\end{array}\right\} \\
& =\frac{1}{\sin ^{2} \omega^{\prime}}\left\{\begin{array}{l}
a^{\prime}-2 h^{\prime} \cos \omega^{\prime}+b^{\prime} \\
-\cos 2 \theta\left(a^{\prime} \cos \overline{2 \omega^{\prime}+2 \alpha^{\prime}}-2 h^{\prime} \cos \overline{\omega^{\prime}+2 \alpha^{\prime}}+b^{\prime} \cos 2 \alpha^{\prime}\right) \\
-\sin 2 \theta\left(a^{\prime} \sin \overline{2 \omega^{\prime}+2 a^{\prime}}-2 h^{\prime} \sin \overline{\omega^{\prime}+2 \alpha^{\prime}}+l^{\prime} \sin 2 a^{\prime}\right)
\end{array}\right\} .
\end{aligned}
$$

Now if

$$
l+m \cos \phi+n \sin \phi=l^{\prime}+m^{\prime} \cos \phi+n^{\prime} \sin \phi
$$

holds for all values of $\phi$ then must

$$
\begin{aligned}
& l=l^{\prime}, \\
& m=m^{\prime}, \\
& n=n^{\prime} \text { and } m^{2}+n^{\prime \prime}=m^{\prime \prime}+n^{\prime 2} .
\end{aligned}
$$

Hence from above

and

$$
\frac{a-2 h \cos \omega+b}{\sin ^{2} \omega}=\frac{a^{\prime}-2 h^{\prime} \cos \omega^{\prime}+b^{\prime}}{\sin ^{2} \omega^{\prime}}
$$

$$
\begin{aligned}
& \frac{1}{\sin ^{4} \omega}\left\{\begin{array}{c}
(a \cos \overline{2 \omega+2 \alpha}-2 h \cos \overline{(\omega+2 \alpha}+b \cos 2 a)^{2} \\
(a \sin \overline{2 \omega+2 \alpha}-2 h \sin \overline{\omega+2 \alpha}+b \sin 2 \alpha)^{2}
\end{array}\right\}=\frac{1}{\sin ^{4} \omega^{\prime}}\left\{\begin{array}{c}
\text { a similar } \\
\text { expression }
\end{array}\right\} ; \\
& \therefore \quad \frac{1}{\sin ^{4} \omega}\left\{\begin{array}{l}
a^{2}+4 h^{2}+b^{2} \\
-4 h \cos \omega(a+b)+2 a b \cos 2 \omega
\end{array}\right\}=\frac{1}{\sin ^{4} \omega^{\prime}}\left\{\begin{array}{c}
\text { a similar } \\
\text { expression }
\end{array}\right\} \text { : } \\
& \therefore \quad \frac{1}{\sin ^{4} \omega}\left\{\begin{array}{c}
(a-2 h \cos \omega+b)^{2} \\
+4\left(h^{\prime \prime}-a b\right) \sin ^{2} \omega
\end{array}\right\}=\frac{1}{\sin ^{4} \omega^{\prime}}\left\{\begin{array}{c}
\left(a^{\prime}-2 h^{\prime} \cos \omega^{\prime}+b^{\prime}\right)^{2} \\
+4\left(h^{\prime}-a^{\prime} b^{\prime}\right) \sin ^{2} \omega^{\prime}
\end{array}\right\} \text {. }
\end{aligned}
$$

Take from both sides the squares of the equals (1)

$$
\begin{aligned}
& \text { i.e., }\left(\frac{a-2 h \cos \omega+b}{\sin ^{2} \omega}\right)^{2}=\left(\frac{a^{\prime}-2 h^{\prime} \cos \omega^{\prime}+b^{\prime}}{\sin ^{2} \omega^{\prime}}\right)^{2} \text {, } \\
& \therefore \quad \frac{4\left(l^{2}-a b\right) \sin ^{2 \prime}()}{\sin ^{4}(\omega)}=\frac{1\left(h^{\prime \prime 2}-a^{\prime} b^{\prime}\right) \sin ^{2} \omega^{\prime}}{\sin ^{4}\left(\omega^{\prime}\right.} ; \\
& \therefore \quad \frac{h^{2}-a b}{\sin ^{2}(1)}=\frac{h^{\prime 2}-a^{\prime} b^{\prime}}{\sin ^{2}\left(\omega^{\prime}\right.} \text {. }
\end{aligned}
$$

